\title{
Bile acids lower triglyceride levels via a pathway involving FXR, SHP, and SREBP-1c
}

\author{
Mitsuhiro Watanabe, ${ }^{1}$ Sander M. Houten, ${ }^{1}$ Li Wang, ${ }^{2}$ Antonio Moschetta, ${ }^{3}$ David J. Mangelsdorf, ${ }^{3}$ \\ Richard A. Heyman, ${ }^{4}$ David D. Moore, ${ }^{2}$ and Johan Auwerx ${ }^{1,5}$
}

\begin{abstract}
1Institut de Génétique et Biologie Moléculaire et Cellulaire (IGBMC), CNRS/INSERM/ULP, Illkirch, France. 2Department of Molecular and Cellular Biology, Baylor College of Medicine, Houston, Texas, USA. ${ }^{3}$ Howard Hughes Medical Institute and Department of Pharmacology, University of Texas Southwestern Medical Center, Dallas, Texas, USA. ${ }^{4}$ X-ceptor Therapeutics, San Diego, California, USA. ${ }^{5}$ Institut Clinique de la Souris, Illkirch, France.
\end{abstract}

\begin{abstract}
We explored the effects of bile acids on triglyceride (TG) homeostasis using a combination of molecular, cellular, and animal models. Cholic acid (CA) prevents hepatic TG accumulation, VLDL secretion, and elevated serum TG in mouse models of hypertriglyceridemia. At the molecular level, CA decreases hepatic expression of SREBP-1c and its lipogenic target genes. Through the use of mouse mutants for the short heterodimer partner (SHP) and liver X receptor (LXR) $\alpha$ and $\beta$, we demonstrate the critical dependence of the reduction of SREBP-1c expression by either natural or synthetic farnesoid X receptor (FXR) agonists on both SHP and LXR $\alpha$ and LXR $\beta$. These results suggest that strategies aimed at increasing FXR activity and the repressive effects of SHP should be explored to correct hypertriglyceridemia.
\end{abstract}

\section{Introduction}

Hypertriglyceridemia is a strong predictor of coronary heart disease. This is mainly attributed to the inverse relationship between serum triglycerides (TGs) and HDL cholesterol, since low levels of HDL increase the risk of vascular disease. However, several recent findings have provided compelling evidence that TGs are also an independent risk factor $(1,2)$. In most cases, hypertriglyceridemia is secondary to a westernized life-style, which is characterized by a lack of physical exercise and increased caloric intake. Such a life-style is associated with visceral fat accumulation, insulin resistance, and hypertriglyceridemia (type IIb and type IV hyperlipoproteinemia), as a consequence of an increased production of VLDL by the liver.

The balance between TG production and clearance determines the circulating TG levels. De novo synthesis of fatty acid and TGs from dietary carbohydrates occurs mainly in the liver, where the TGs are packaged as VLDLs. VLDL TGs are cleared from the circulation through the action of lipases, which hydrolyze TGs into FFAs that can be used as a source of energy in muscle and heart, stored in adipose tissue, or taken up by the liver. Additional factors such as ApoC-II and angiopoietin-like protein 3 (ANGPTL3) can influence the action of lipases. Liver TG production is mainly determined by the fatty acid synthesis rate, which is controlled to a large extent at the level of transcription by both PPAR $\alpha$ (NR1C1) (3), which stimulates fatty acid $\beta$-oxidation, and SREBP-1c (4), which controls fatty acid synthesis. SREBPs are basic helix-loop-helix leucine zipper transcription factors that are mainly known for their control of genes that govern biosynthesis

Nonstandard abbreviations used: acetyl-CoA carboxylase (ACC); acetyl-CoA synthetase (AceCS); angiopoietin-like protein 3 (ANGPTL3); carnitine palmitoyltransferase I (CPT-I); chenodeoxycholic acid (CDCA); cholesterol $7 \alpha$-hydroxylase (CYP7A1); cholic acid (CA); farnesoid X receptor (FXR); fatty acid synthase (FAS); LDL receptor (LDL-R); liver receptor homolog-1 (LRH-1); liver receptor homolog-1 response element (LRH-1RE); liver X receptor (LXR); liver X receptor response element (LXRRE); long-chain acyl-CoA dehydrogenase (LCAD); malic enzyme (ME); medium-chain acyl-CoA dehydrogenase (MCAD); retinoid X receptor (RXR); short heterodimer partner (SHP); stearoyl-CoA desaturase-1 (SCD-1); triglyceride (TG). Conflict of interest: The authors have declared that no conflict of interest exists.

Citation for this article: J. Clin. Invest. 113:1408-1418 (2004).

doi:10.1172/JCI200421025 of cholesterol and its receptor-mediated uptake from $\operatorname{LDL}(4,5)$. SREBPs, and in particular SREBP-1, also control the expression of genes involved in lipogenesis, such as acetyl-CoA carboxylase (ACC), fatty acid synthase (FAS), acetyl-CoA synthetase (AceCS), and glycerol-3-phosphate acyltransferase. In addition, SREBPs can induce the expression of ATP-citrate lyase, malic enzyme (ME), glucose-6-phosphate dehydrogenase, and 6-phosphogluconate dehydrogenase $(4,5)$. These enzymes generate NADPH and cytosolic acetyl-CoA, which are essential for lipogenesis. SREBPs also activate stearoyl-CoA desaturase-1 (SCD-1), an enzyme involved in the synthesis of monounsaturated fatty acids $(4,5)$. The lipogenic role for SREBP-1 was underscored in vivo by the elevation of fatty acid biosynthesis and TG levels in the livers of transgenic animals, which overexpressed either the mature form of SREBP-1a (6) or SREBP-1c (7). Finally, expression of SREBP-1c is enhanced by insulin, which explains the classic ability of insulin to enhance the conversion of glucose to fatty acid $(8,9)$. Recently, the basal transcription of SREBP-1c was shown to depend on liver $X$ receptor (LXR $\alpha, N R 1 H 3$, and LXR $\beta$, NR1H2). Furthermore, the activation of LXR induces SREBP-1c expression, resulting in a rise in VLDL TGs $(10,11)$.

Bile acids have long been known to affect TG homeostasis. In humans, bile acid-binding resins induce the production of VLDL TGs (12-14), whereas treatment of cholesterol gallstones with the bile acid chenodeoxycholic acid (CDCA) has been shown to reduce hypertriglyceridemia $(12,15,16)$. The mechanism underlying this reciprocal relationship between bile acid biosynthesis and TG production has remained elusive, but two possible explanations have been postulated. At the transcriptional level, bile acids, which are the endogenous ligands of the farnesoid $\mathrm{X}$ receptor (FXR, NR1H4), activate the transcription of several genes that could modulate TG levels, such as the atypical nuclear receptor short heterodimer partner (SHP, NR0B2) $(17,18), \operatorname{PPAR} \alpha(19)$, and ApoC-II (20). Alternatively, at the metabolite level, a reduction in bile acid biosynthesis could increase hepatic cholesterol and oxysterol levels, which will influence the function of the lipogenic SREBP-1c by attenuating its processing and activation. This could lead to decreased TG production $(21,22)$. Here, we 
provide evidence in support of the first hypothesis and show that bile acids, by activating FXR, induce the expression of SHP. SHP then interferes with SREBP-1c expression by inhibiting the activity of LXR and eventually other transcription factors that stimulate SREBP-1c expression.

\section{Methods}

Materials. Cholesterol, 22(R)-hydroxycholesterol, 25-hydroxycholesterol, CA, and CDCA were obtained from Sigma-Aldrich (St. Quentin Fallavier, France).

Plasmids. pCMX-SHP was obtained by insertion of a PCR product corresponding to the mouse SHP cDNA into the PCMX vector. pCMX-liver receptor homolog-1 (pCMX-LRH-1) was produced by insertion of a PCR product corresponding to the mouse LRH-1 cDNA into pCMX. The $\mathrm{PCMX}-\mathrm{LXR} \alpha$ expression vector was as described (10); the pSG5-retinoid X receptor $\alpha$ (pSG5-RXR $\alpha)$ expression vector was a gift of $\mathrm{P}$. Chambon (Institut Clinique de la Souris, Illkirch, France). The SREBP-1c promoter luciferase reporter plasmids were generated by PCR amplification of promoter fragments corresponding to sequences located between -1070 to -51 of the mouse SREBP-1c gene. The PCR product was ligated into the pGL3 basic vector (Promega, Madison, Wisconsin, USA). The reverse primer used for each promoter construct is 5'-CTTCCGCGCCGATTTCACCTG-3'. The different forward primers used for each construct are as follows: pSREBP-1c1070Luc (5'-ACCCCTCAGACTGTGTGAGT-3'), pSREBP-1c571-Luc (5'-CTA GCTAGATGACCCTGCACCACCAA-3'), pSREBP-1c327Luc (5'-TTGCCTGTGCGGCAGGGGTTGGGACGA-3'), pSREBP1c276-Luc (5'-CGCGCTGGCGCAGACGCGGTTAAA-3'), and pSREBP-1c151-Luc (5'-CTGCTGATTGGCCATGTGCGCTCA-3'). The primers were tailed with either a $K p n I$ site (forward) or a $B g l I I$ site (reverse). The LXR response elements in pSREBP-1c324-Luc were mutated for promoter analysis using the Quick Change SiteDirected Mutagenesis Kit (Stratagene, La Jolla, California, USA). All constructs were verified by sequence analysis.

Animals. Male C57BL/6J, KK- $A^{y}$, and $o b / o b$ (C57BL/6J background) mice, 6-10 weeks of age, were obtained from Elevage Janvier (Le Genest St. Isle, France), CLEA Japan Inc. (Tokyo, Japan), and The Jackson Laboratory (Bar Harbor, Maine, USA), respectively. All mice were maintained in a temperature-controlled $\left(23^{\circ} \mathrm{C}\right)$ facility with a 12-hour light/dark cycle and were given free access to food and water. The study protocols were approved by the institutional review boards. The body weight and food intake were measured every second day. The control and high-fat diet was obtained from UAR (Villemoisson sur Orge, France). The control diet (EQ12310) contained $16.8 \%$ protein, $73.5 \%$ carbohydrate, and $4.8 \%$ fat, whereas the high-fat diet (EQ/D12309) contained $23.0 \%$ protein, $35.5 \%$ carbohydrate, and $35.9 \%$ fat. For treatment with bile acids, mice were fed diets with $0.5 \%(\mathrm{w} / \mathrm{w})$ CA. The mice were fasted 4 hours before harvesting blood for subsequent lipid measurements, and tissues for RNA isolation, lipid measurements, and histology. Male $\mathrm{SHP}^{-/-}$mice and littermate $\mathrm{SHP}^{+/+}$controls (C57/BL6-A129/SvJ mixed strain) of 8 weeks were fed the CA diet as just described or gavaged with GW4064 dissolved in corn oil at $30 \mathrm{mg} / \mathrm{kg}$ (23). Male wild-type and $L X R \alpha / \beta^{-/-}$mice (C57/BL6-A129/SvJ mixed strain; ref. 10) 10-14 weeks old were fed a powdered chow diet (Harlan Teklad 7001, Madison, Wisconsin, USA) and, where indicated, mixed with $0.5 \%$ CA. A T0901317 suspension prepared in a 1\% carboxymethylcellulose solution was used for gavage at $30 \mathrm{mg} / \mathrm{kg}$. KK- $A^{y}$ and $o b / o b$ mice on the control diet were treated daily by intraperitoneal injection for 1 week with GW4064 prepared in a 1\% carboxymethylcellulose solution at $10 \mathrm{mg} / \mathrm{kg}$.

Lipid measurements and liver function tests. Serum total cholesterol, TGs, and lipoprotein cholesterol and TG profiles were measured as described $(24,25)$. For measurement of liver TGs and cholesterol content, a liver was homogenized in chloroform/ methanol $(2: 1 \mathrm{v} / \mathrm{v})$ using a Polytron tissue grinder (Kinematica AG, Luzern, Switzerland). Lipid extracts were prepared by the classical Folch method. Extracts were dried under $\mathrm{N}_{2}$ flow and resuspended in isopropanol. For in vivo measurements of VLDL TG production, mice were injected with Tyloxapol (SigmaAldrich) (500 $\mu \mathrm{g} / \mathrm{g}$ body weight), after which blood samples were taken at the indicated time points (26).

Cell culture, transient transfection, and luciferase assays. The rat hepatoma cell line McA-RH7777 was obtained from ATCC (Manassas, VA, USA). Cells were grown at $37^{\circ} \mathrm{C}$ in a humidified atmosphere of $5 \% \mathrm{CO}_{2} / 95 \%$ air. Transfections were performed in 96-well plates using Lipofectamine 2000 (Invitrogen, Cergy Pontoise, France). Each well contained $64 \mathrm{ng}$ luciferase reporter and $4 \mathrm{ng}$ of $\beta$-galactosidase expression plasmid. When indicated, we transfected $16 \mathrm{ng} \mathrm{RXR} \alpha, 16$ ng LXR $\alpha, 16$ ng LRH-1, 32 ng SHP expression plasmid, or the corresponding empty expression vectors. After 6 hours of incubation with the DNA-lipofectamine complexes, the transfection medium was exchanged for medium with or without the indicated ligands. Ligands for LXR and RXR were dissolved in ethanol or DMSO and added to the cells in DMEM supplemented with 10\% lipoproteindeficient serum. To suppress SREBP-1c activation by processing, leading to feed-forward induction of SREBP-1c expression, the medium used for the SREBP-1c promoter study was also supplemented with $10 \mu \mathrm{g} / \mathrm{ml}$ cholesterol and $1 \mu \mathrm{g} / \mathrm{ml} 25$-hydroxycholesterol. Luciferase measurements were normalized to $\beta$-galactosidase activity.

Hepatocytes. Hepatocytes were isolated from wild-type male mice 8-10 weeks old. Animals were anesthetized with $\mathrm{CO}_{2}$ and livers were perfused with $40 \mathrm{ml}$ Liver Perfusion Medium (Invitrogen) followed by $30 \mathrm{ml}$ Liver Digestion Medium (Invitrogen), both at a flow rate of $5 \mathrm{ml} / \mathrm{min}$. Isolated hepatocytes were resuspended in DMEM supplemented with $20 \%$ (v/v) FCS, 100 units/ml penicillin, and 100 units $/ \mathrm{ml}$ streptomycin, and placed in plates coated with collagen IV at a density of $1 \times 10^{5} \mathrm{cells} / \mathrm{cm}^{2}$. After a 4-hour incubation at $37^{\circ} \mathrm{C}$ in an atmosphere of $5 \% \mathrm{CO}_{2}$, the medium was changed to DMEM supplemented with $10 \%$ (v/v) FCS, 100 units/ $\mathrm{ml}$ penicillin, 100 units $/ \mathrm{ml}$ streptomycin, $0.5 \mu \mathrm{M}$ dexamethasone (Sigma-Aldrich), and $0.4 \mu \mathrm{M}$ insulin (Sigma-Aldrich). After 16 hours, the medium was changed into medium supplemented with CDCA and ligands for LXR and RXR. After an additional 18-hour incubation, cells were harvested for RNA isolation.

Expression-level analysis. Total RNA was extracted from frozen tissue samples or cells using the RNeasy kit (QIAGEN, Courtaboeuf, France). cDNA was synthesized from total RNA with the SuperScript First-Strand Synthesis System (Invitrogen) and random hexamer primers. The real-time PCR measurement of individual cDNAs was performed using SYBR green dye to measure duplex DNA formation with the LightCycler System (Roche Diagnostics, Meylan, France). The sequences of the primer sets used are available online at http://www-igbmc.u-strasbg.fr/Departments/Dep_V/ Dep_VA/Publi/Paper.html.

Statistical analysis. Values were reported as mean \pm SE. Statistical differences were determined by either a Student's $t$ test or an ANOVA followed by a Bonferroni or Dunnett post test. Statistical significance is displayed as $*(P<0.05)$ or $* *(P<0.01)$. 
A
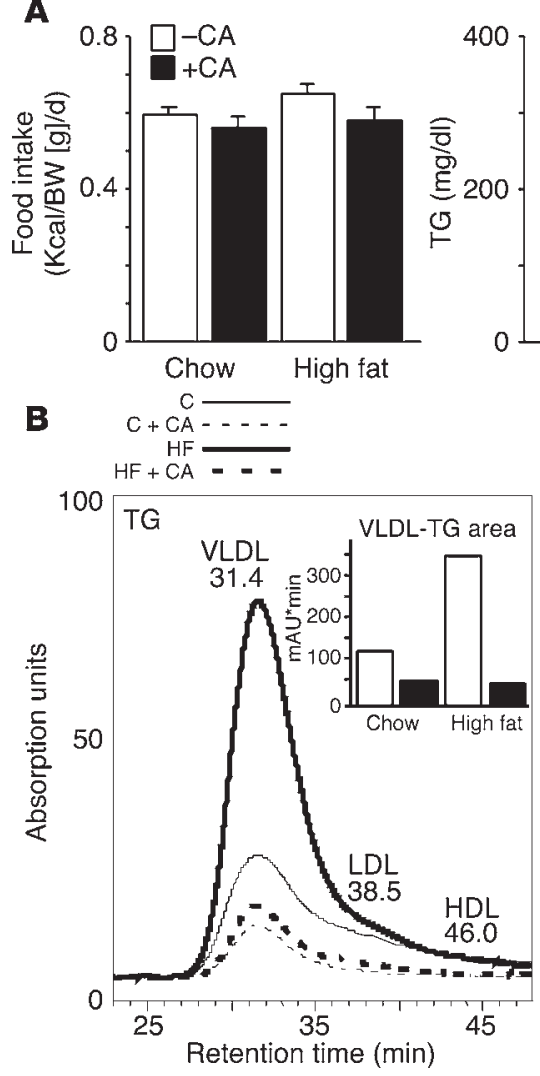

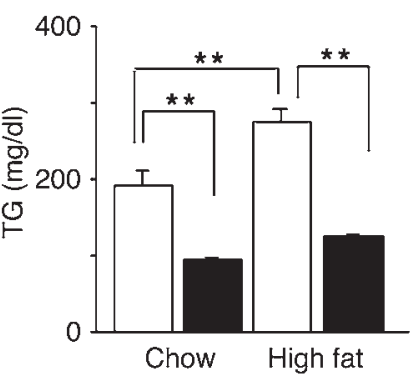

Chow fat

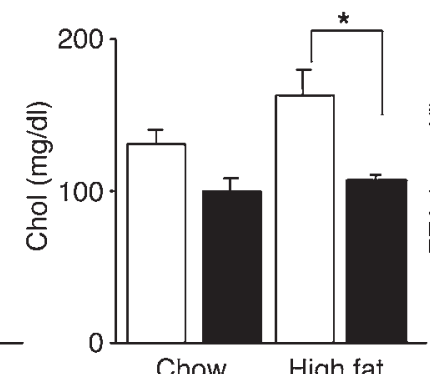

High fat
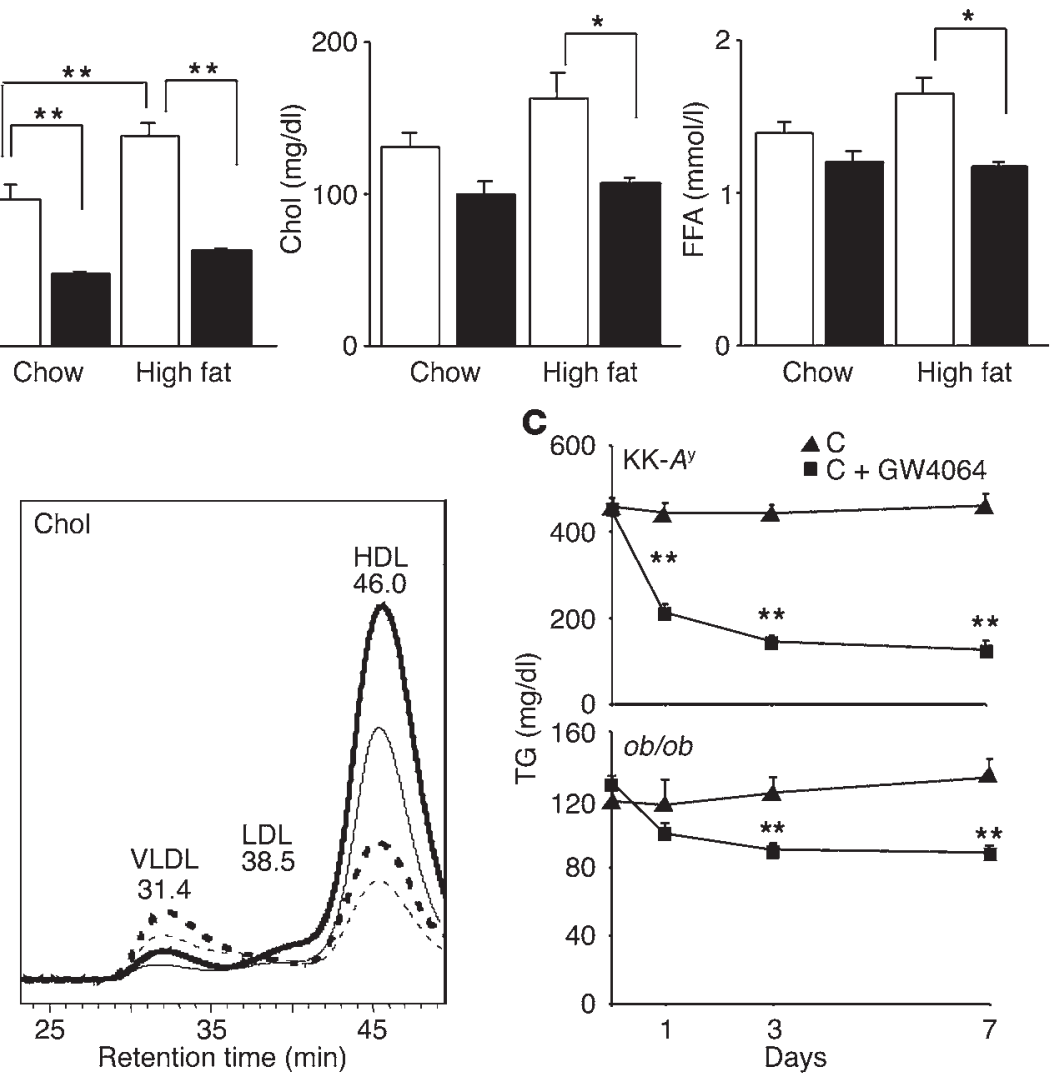

Figure 1

CA lowers serum TGs in KK-A $A^{y}$ mice. (A) Food intake of KK- $A^{y}$ mice during 1 week on the diets as described in the figure. Serum levels of TGs, total cholesterol (Chol), and FFA in KK- $A^{y}$ mice after 1 week on the different diets (age 7 weeks, $n=5$ ). Kcal, kilocalories; BW, body weight. (B) $\mathrm{TG}$ and cholesterol lipoprotein profiles after size-exclusion chromatography of serum pools from five animals. The quantification of the VLDL TGs is shown in an inset. (C) Serum TGs in KK- $A^{y}$ (top, age 12 weeks, $n=4$ ) and ob/ob (bottom, age 12 weeks, $n=4$ ) mice after $0,1,3$, and 7 days of treatment with the synthetic FXR agonist GW4064. ${ }^{*} P<0.05 ;{ }^{* *} P<0.01$ throughout the figures.

\section{Results}

Cholic acid lowers serum TGs in KK-Ay mice. To study the TG-lowering effect of bile acids in vivo, we first explored the effect of feeding CA on TG homeostasis in $\mathrm{KK}-A^{y}$ mice. $\mathrm{KK}$ is an inbred strain that develops type 2 diabetes mellitus with only mild obesity, even after maturity (27). Introduction of the lethal yellow mutation in the agouti gene $\left(A^{y}\right)(28)$ causes overt type 2 diabetes and massive obesity with a relatively late onset (29). These KK- $A y$ mice are characterized by a severe prolonged hyperinsulinemia, hyperglycemia, and hyperlipidemia. Under basal conditions the elevation of TGs is only moderate $(192 \pm 16 \mathrm{mg} / \mathrm{dl})$, but can be further increased by a high-fat diet $(273 \pm 14 \mathrm{mg} / \mathrm{dl}$, Figure $1 \mathrm{~A})$. This elevation of serum TG levels on a high-fat diet is not observed in normal C57BL/6J mice (data not shown and ref. 30), making the KK- $A^{y}$ mice a unique model to study diet-induced hypertriglyceridemia. In addition, the elevation of serum TG is progessive and dependent on the age of the KK- $A^{y}$ mice. At 7 weeks of age the hypertriglyceridemia is moderate $(192 \pm 16 \mathrm{mg} / \mathrm{dl}$, Figure $1 \mathrm{~A})$, whereas at 12 weeks of age, it is severe $(453 \pm 24 \mathrm{mg} / \mathrm{dl}$, Figure $1 \mathrm{C})$.

Male KK- $A^{y}$ mice were given normal chow or high-fat diets with or without $0.5 \%$ CA for 1 week. The CA-containing diets were well tolerated, and food intake was not affected during a 1-week (Figure 1A) and a 3-week study (not shown). Interestingly, CA robustly lowered circulating TG levels in both $\mathrm{KK}-A^{y}$ mice on a normal-chow (51\%) and on a high-fat diet (65\%, Figure 1A). Total plasma cholesterol was also decreased $(24 \%$ on a normal-chow diet and $35 \%$ on a high-fat diet, Figure 1A). CA feeding decreased plasma FFAs, particularly in mice on the highfat diet (Figure 1A). High-performance liquid chromatography analysis of lipoproteins demonstrated that CA feeding decreased plasma TGs primarily by decreasing VLDL TGs, and decreased cholesterol primarily by decreasing HDL cholesterol. CA feeding increased LDL cholesterol levels (Figure 1B).

To verify whether the observed effects of CA were mediated through FXR, we treated chow-fed KK- $A^{y}$ and $o b / o b$ mice during 1 week with the synthetic FXR agonist GW4064. GW4064 potently lowered serum TG levels in both the KK- $A^{y}$ and $o b / o b$ mice (Figure $1 C)$, suggesting that the observed effects are mediated by FXR. In addition, this experiment shows that the TG-lowering effect of FXR activation is not restricted to KK- $A^{y}$ mice, a model for dietinduced hypertriglyceridemia, but also occurs in another model of hypertriglyceridemia, the $o b / o b$ mouse.

$C A$ lowers hepatic TG levels. Administration of CA to KK- $A^{y}$ mice for 3 weeks also changed liver morphology. Livers of chow-fed KK- $A^{y}$ mice have a pale color, suggestive of increased lipid storage. Livers of animals that were treated with CA were less pale and had a more normal reddish appearance (Figure $2 \mathrm{~A}$ ). This effect of bile acids on hepatosteatosis was more pronounced in mice fed high-fat diets, 
A
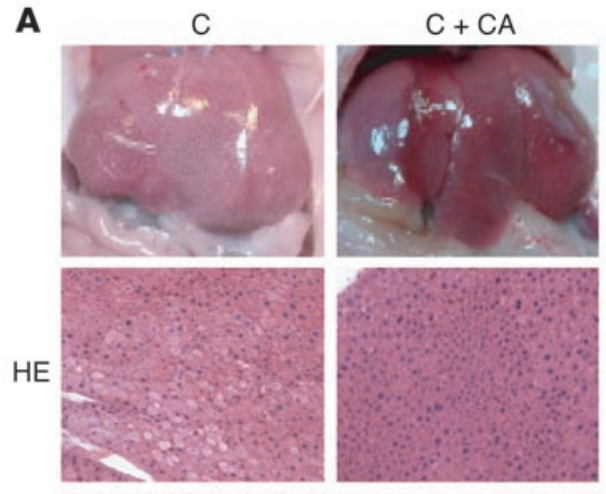

OR

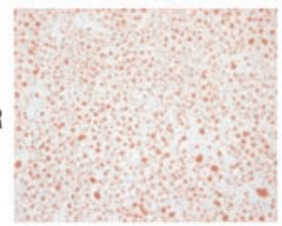

B

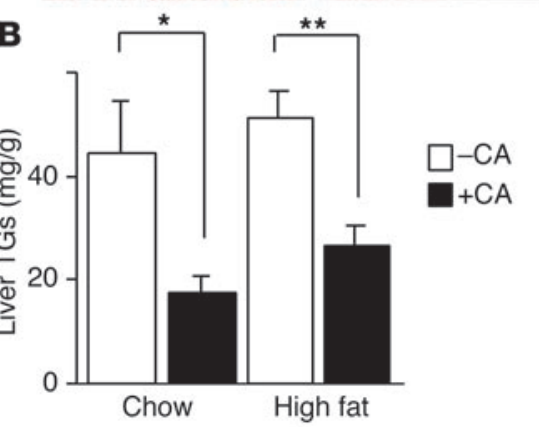

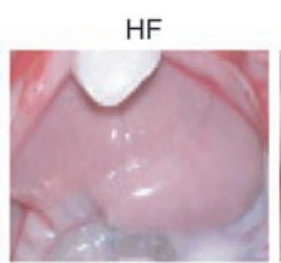
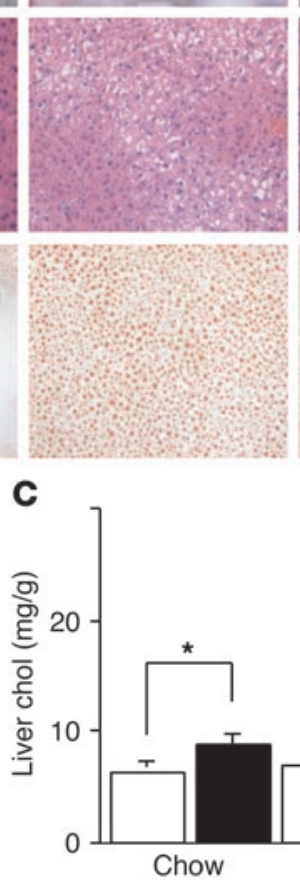

$\mathrm{HF}+\mathrm{CA}$
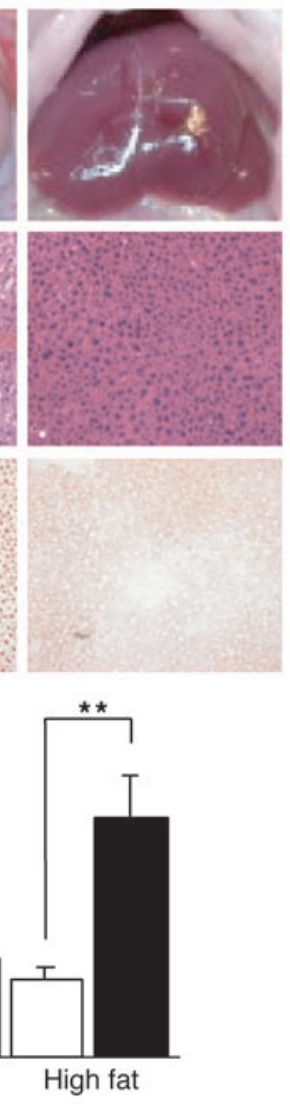

D
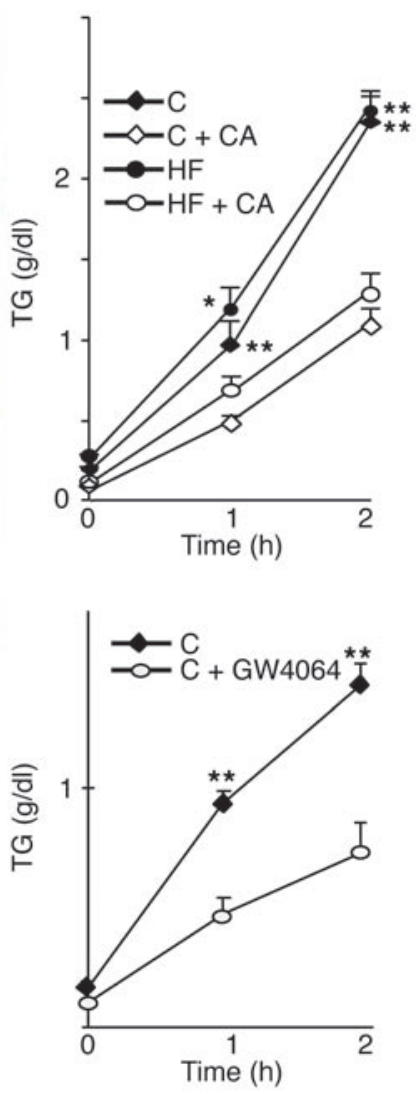

Figure 2

CA lowers hepatic TGs in KK-Ay mice. (A) Liver morphology, H\&E-stained liver sections (HE), and Oil Red O-stained liver sections (OR) in animals treated with the indicated diets for 3 weeks. When sacrificed, the mice were 9 weeks old. (B) Liver TG and (C) cholesterol content after 3 weeks on the different diets (age 9 weeks, $n=5$ ). (D) In vivo VLDL secretion from the livers of mice on the different diets (age 7 weeks, $n=4)$ or after treatment with GW4064 (age 12 weeks, $n=4$ ). C, control diet; C + CA, control diet with CA; HF, high-fat diet; HF + CA, high-fat diet with CA.

which showed an impressive return to a normal morphology. H\&Estained sections of the livers of animals on the high-fat diet with CA showed much lower levels of unstained inclusions (Figure 2A). Staining of these liver sections with Oil Red O (Sigma-Aldrich) demonstrated that CA-treated animals accumulated less neutral lipids, an effect coherent with both gross morphological appearance and H\&E staining (Figure 2A). Consistent with the morphological appearance, livers of CA-treated animals contained significantly lower amounts of TGs in animals on chow or high-fat diet (Figure 2B). In contrast, CA feeding increased hepatic cholesterol, particularly in the animals fed high-fat diets (Figure 2C).

To assess whether the decrease in liver TG content is associated with decreased export, we measured VLDL production in KK- $A^{y}$ mice after 1 week of CA feeding. CA feeding significantly decreased liver VLDL production on both a chow and high-fat diet (Figure 2D). In a similar experiment, a 1-week administration of GW4064 also significantly lowered VLDL secretion in KK- $A^{y}$ mice (Figure $2 \mathrm{D})$, again suggesting that this effect is mediated via FXR.

$C A$ decreases expression of SREBP-1c and other lipogenic genes. To better understand the molecular mechanism underlying the TG-lowering effect of bile acids, we used quantitative RT-PCR to measure hepatic mRNA levels of several important proteins involved in lipid homeostasis in $\mathrm{KK}-A^{y}$ and $\mathrm{C} 57 \mathrm{BL} / 6 \mathrm{~J}$ mice after
1 and 7 days of CA supplementation of a chow diet (Table 1). No major changes were observed in the expression of several transcription factors involved in liver lipid and bile acid homeostasis, such as LXR $\alpha$, LRH-1 (NR5A2), FXR, and SREBP-2. Expression of the LDL receptor (LDL-R) and genes involved in cholesterol biosynthesis had a tendency to decrease, whereas genes involved in encoding cholesterol transport proteins (ABCA1 and ABCG5) were slightly increased, although these differences never reached statistical significance. Expression of genes encoding enzymes involved in fatty acid and TG biosynthesis, such as AceCS, ME, and SCD-1, was significantly reduced by CA feeding. This reduction is strongest after 1 day of treatment and is mitigated somewhat after 7 days of treatment. To verify whether an increase in fatty acid $\beta$-oxidation contributed to the TG-lowering effects in our studies, we measured the hepatic expression levels of liver carnitine palmitoyltransferase I (CPT-I), medium-chain acyl-CoA dehydrogenase (MCAD), and long-chain acyl-CoA dehydrogenase (LCAD). In C57BL/6J mice, the expression of MCAD and LCAD was significantly decreased after 1 and 7 days of treatment with CA. Interestingly, in KK- $A^{y}$ mice there was no change in the expression of these genes. Thus increased expression of genes involved in $\beta$-oxidation of fatty acids is not responsible for the decrease in serum TG. Aside from these differences in 


\section{Table 1}

CA decreases expression of SREBP-1c and other lipogenic genes

\begin{tabular}{|c|c|c|c|c|c|c|}
\hline & \multicolumn{3}{|c|}{ C57BL/6J $(n=4)$} & \multicolumn{3}{|c|}{$\mathrm{KK}-A^{y}(n=5)$} \\
\hline & Control diet & CA 1 day & CA 7 days & Control diet & CA 1 day & CA 7 days \\
\hline \multicolumn{7}{|l|}{ SREBP pathway } \\
\hline $\begin{array}{l}\text { SREBP-1C } \\
\text { SREBP-2 }\end{array}$ & $\begin{array}{l}1.00 \pm 0.12 \\
1.00 \pm 0.11\end{array}$ & $\begin{array}{l}0.53 \pm 0.05^{A} \\
0.94 \pm 0.13\end{array}$ & $\begin{array}{l}0.78 \pm 0.09 \\
0.82 \pm 0.10\end{array}$ & $\begin{array}{l}1.00 \pm 0.19 \\
1.00 \pm 0.18\end{array}$ & $\begin{array}{l}0.49 \pm 0.09 A \\
0.76 \pm 0.11\end{array}$ & $\begin{array}{l}0.73 \pm 0.06 \\
0.77 \pm 0.28\end{array}$ \\
\hline \multicolumn{7}{|l|}{ Nuclear receptors } \\
\hline $\begin{array}{l}\operatorname{LXR} \alpha \\
\text { FXR } \\
\text { LRH-1 } \\
\text { SHP }\end{array}$ & $\begin{array}{l}1.00 \pm 0.09 \\
1.00 \pm 0.20 \\
1.00 \pm 0.14 \\
1.00 \pm 0.33\end{array}$ & $\begin{array}{l}1.23 \pm 0.15 \\
0.76 \pm 0.19 \\
0.74 \pm 0.20 \\
2.30 \pm 0.37^{A}\end{array}$ & $\begin{array}{l}1.02 \pm 0.07 \\
0.76 \pm 0.13 \\
0.74 \pm 0.12 \\
2.34 \pm 0.43^{A}\end{array}$ & $\begin{array}{l}1.00 \pm 0.06 \\
1.00 \pm 0.14 \\
1.00 \pm 0.25 \\
1.00 \pm 0.19\end{array}$ & $\begin{array}{l}0.82 \pm 0.06 \\
0.77 \pm 0.16 \\
0.77 \pm 0.23 \\
3.84 \pm 0.42^{B}\end{array}$ & $\begin{array}{l}0.98 \pm 0.31 \\
1.03 \pm 0.29 \\
0.78 \pm 0.27 \\
4.34 \pm 0.97^{A}\end{array}$ \\
\hline \multicolumn{7}{|c|}{ Fatty acid and TG biosynthesis } \\
\hline $\begin{array}{l}\text { FAS } \\
\text { AceCS } \\
\text { ACC } \\
\text { SCD-1 } \\
\text { ME }\end{array}$ & $\begin{array}{l}1.00 \pm 0.16 \\
1.00 \pm 0.14 \\
1.00 \pm 0.15 \\
1.00 \pm 0.15 \\
1.00 \pm 0.03\end{array}$ & $\begin{array}{l}0.48 \pm 0.07 \\
0.39 \pm 0.09^{B} \\
1.26 \pm 0.27 \\
0.33 \pm 0.10^{A} \\
0.60 \pm 0.15^{A}\end{array}$ & $\begin{array}{l}1.37 \pm 0.46 \\
0.56 \pm 0.02^{\mathrm{A}} \\
1.23 \pm 0.31 \\
0.98 \pm 0.19 \\
0.51 \pm 0.08^{\mathrm{A}}\end{array}$ & $\begin{array}{l}1.00 \pm 0.21 \\
1.00 \pm 0.14 \\
1.00 \pm 0.16 \\
1.00 \pm 0.05 \\
1.00 \pm 0.06\end{array}$ & $\begin{array}{l}0.47 \pm 0.12 \\
0.52 \pm 0.10^{A} \\
0.61 \pm 0.10^{-1} \\
0.36 \pm 0.08^{B} \\
0.45 \pm 0.05^{B}\end{array}$ & $\begin{array}{l}1.26 \pm 0.37 \\
0.66 \pm 0.12 \\
1.01 \pm 0.17 \\
0.83 \pm 0.13 \\
0.48 \pm 0.06 \mathrm{~B}\end{array}$ \\
\hline \multicolumn{7}{|c|}{ Cholesterol homeostasis and bile acid biosynthesis } \\
\hline $\begin{array}{l}\text { HMG-CoA Synthase } \\
\text { HMG-CoA Reductase } \\
\text { LDL receptor } \\
\text { CYP7A1 } \\
\text { ABCA1 } \\
\text { ABCG5 }\end{array}$ & $\begin{array}{l}1.00 \pm 0.25 \\
1.00 \pm 0.26 \\
1.00 \pm 0.07 \\
1.00 \pm 0.26 \\
1.00 \pm 0.26 \\
1.00 \pm 0.20\end{array}$ & $\begin{array}{l}0.39 \pm 0.04 \\
0.79 \pm 0.10 \\
0.66 \pm 0.11 \\
0.07 \pm 0.03^{B} \\
1.04 \pm 0.24 \\
0.82 \pm 0.26\end{array}$ & $\begin{array}{l}0.35 \pm 0.14^{A} \\
0.55 \pm 0.17 \\
0.43 \pm 0.09^{B} \\
0.07 \pm 0.04^{B} \\
1.52 \pm 0.19 \\
1.47 \pm 0.30\end{array}$ & $\begin{array}{l}1.00 \pm 0.28 \\
1.00 \pm 0.13 \\
1.00 \pm 0.11 \\
1.00 \pm 0.30 \\
\text { nd } \\
\text { nd }\end{array}$ & $\begin{array}{l}0.45 \pm 0.11 \\
0.71 \pm 0.09 \\
0.59 \pm 0.06 \\
0.06 \pm 0.03^{A} \\
\text { nd } \\
\text { nd }\end{array}$ & $\begin{array}{l}0.56 \pm 0.12 \\
0.98 \pm 0.11 \\
1.21 \pm 0.22 \\
0.01 \pm 0.01^{\mathrm{A}} \\
\text { nd } \\
\text { nd }\end{array}$ \\
\hline \multicolumn{7}{|l|}{ Fatty acid $\beta$-oxidation } \\
\hline $\begin{array}{l}\text { Liver CPT-I } \\
\text { MCAD } \\
\text { LCAD }\end{array}$ & $\begin{array}{l}1.00 \pm 0.10 \\
1.00 \pm 0.09 \\
1.00 \pm 0.10\end{array}$ & $\begin{array}{l}0.66 \pm 0.22 \\
0.63 \pm 0.12^{A} \\
0.41 \pm 0.16^{A}\end{array}$ & $\begin{array}{l}0.45 \pm 0.08 \\
0.45 \pm 0.08^{B} \\
0.43 \pm 0.10^{A}\end{array}$ & $\begin{array}{l}1.00 \pm 0.15 \\
1.00 \pm 0.12 \\
1.00 \pm 0.09\end{array}$ & $\begin{array}{l}0.69 \pm 0.17 \\
0.95 \pm 0.14 \\
0.93 \pm 0.16\end{array}$ & $\begin{array}{l}1.08 \pm 0.08 \\
1.02 \pm 0.12 \\
0.88 \pm 0.16\end{array}$ \\
\hline
\end{tabular}

At the start of this experiment, the mice were 6 weeks old. The values in the table are relative mRNA levels. The control diet group is set as 1 . nd, not done. ${ }^{A} P<0.05 ;{ }^{B} P<0.01$.

genes involved in $\beta$-oxidation, no major differences were observed between the results obtained in KK- $A^{y}$ and $\mathrm{C} 57 \mathrm{BL} / 6 \mathrm{~J}$ mice.

Interestingly, the changes in expression of genes involved in lipogenesis were paralleled by changes in SREBP-1c expression (Table 1). Furthermore, expression of SHP showed an opposite pattern, with a robust induction upon treatment with CA. SHP is an FXR target gene that represses the activity of several nuclear receptors, including $L R H-1$ and $L X R$, which are essential for the transcription of cholesterol $7 \alpha$-hydroxylase (CYP7A1; see Table 1 ), the rate-limiting enzyme in bile acid biosynthesis. This FXRmediated SHP induction underlies the negative-feedback regulation of bile acid biosynthesis $(17,18,23,31-33)$. The effect of CA on lipogenesis seems to be specific for the liver, since in white adipose tissue, none of the lipogenic genes were lowered in expression in response to CA treatment (data not shown).

The activity of the SREBP-1c promoter is attenuated by bile acids and SHP. We examined the ability of CDCA to lower expression of endogenous SREBP-1c and its target genes in vitro in mouse primary hepatocytes. The SREBP-1c promoter had previously been shown to be regulated by LXR, an effect that contributes to the TG-raising activity of LXR agonists $(10,11)$. We confirmed the induction of endogenous SREBP-1c expression by RXR and LXR ligands and showed that this expression decreased dose-dependently by increasing amounts of CDCA. The expression of the lipogenic target genes of SREBP-1c decreased likewise (Figure
3A). This reduction was most robust for SCD-1, but expression of AceCS and ME was also reduced. SHP expression was increased by the addition of CDCA. These results demonstrate that, both in vivo and in vitro, the expression of endogenous SREBP-1c, as well as the lipogenic enzymes that are regulated by SREBP-1c, is significantly affected by bile acids.

To examine this potential role of bile acids in the regulation of SREBP-1c expression, we cloned the mouse SREBP-1c promoter and generated a luciferase reporter construct (S1, Figure 3C). McARH7777 rat hepatoma cells were cotransfected with this construct and a LXR $\alpha$ expression vector and treated with LXR and RXR agonists in the presence or absence of CA or CDCA. SREBP-1c promoter activity was induced by transfection of LXR $\alpha$ and/or the addition of its ligands. This increase was attenuated when cells were incubated with either of the bile acids (Figure 3B). Furthermore, induction of SREBP-1c promoter activity by LXR and RXR agonists does not depend on the overexpression of LXR, showing that McA-RH7777 cells have endogenous LXR activity.

The effect of CA on the expression of lipogenic genes, as well as the opposite changes in gene expression of SREBP-1c and SHP, prompted us to analyze the contribution of the FXR-SHP cascade to the regulation of SREBP-1c gene expression. We hence generated a number of reporter constructs containing $5^{\prime}$ nested deletions (S1-S5, Figure 3C) or point mutations in the two previously characterized LXR response elements (LXRREs; S6, Figure 3, C and D). 
As expected, SREBP-1c promoter activity is induced by overexpression of the RXR/LXR $\alpha$ heterodimer in rat hepatoma McA-RH7777 cells in the presence of a natural LXR agonist and a synthetic RXR ligand $(10,11,34)$. When the LXRRE sites are mutated or deleted, basal activity of the promoter is markedly reduced (S5 and S6, Figure $3 \mathrm{E})$. It is probable that this effect is conserved between mice and humans, since SREBP-1c expression is also induced by an LXR agonist in human hepatoma cells and primary hepatocytes $(34,35)$. In addition, the two LXRREs are highly conserved between the mouse and the human SREBP-1c promoter (Figure 3D). Transfection of LRH-1 induced SREBP-1c promoter activity and improved the magnitude of induction by ligand-activated LXR, suggesting that LRH-1 acts as a competence factor for LXR as was reported for a number of genes $(17,18,36)$ (Figure 3E). The induction of the SREBP-1c promoter by LRH-1 was lost after deletion of LXRREa (S4, Figure $3 \mathrm{E}$ ). The finding that the promoter region between -327 and -276 contained no consensus LRH-1 response element (LRH-1RE) suggested that the induction might be mediated via LXRREa. Mutation of the $5^{\prime}$ extension of this LXRRE, which does not disrupt the LXRRE consensus sequence itself, did lead to the loss of response to LRH-1 (S7, Figure 3E), which is in line with the suggestion that the LXRREa might mediate the response of SREBP-1c to LRH-1. Interestingly, cotransfection with SHP potently attenuated the induction of the SREBP-1c reporter in the presence of LRH-1, LXR, and RXR (Figure 3E). From this we conclude that SHP regulates the SREBP-1c promoter. Due to technical problems (the loss of basal promoter activity after the mutagenesis of the LXRREs), we cannot attribute this effect with certainty to a particular site in the promoter.

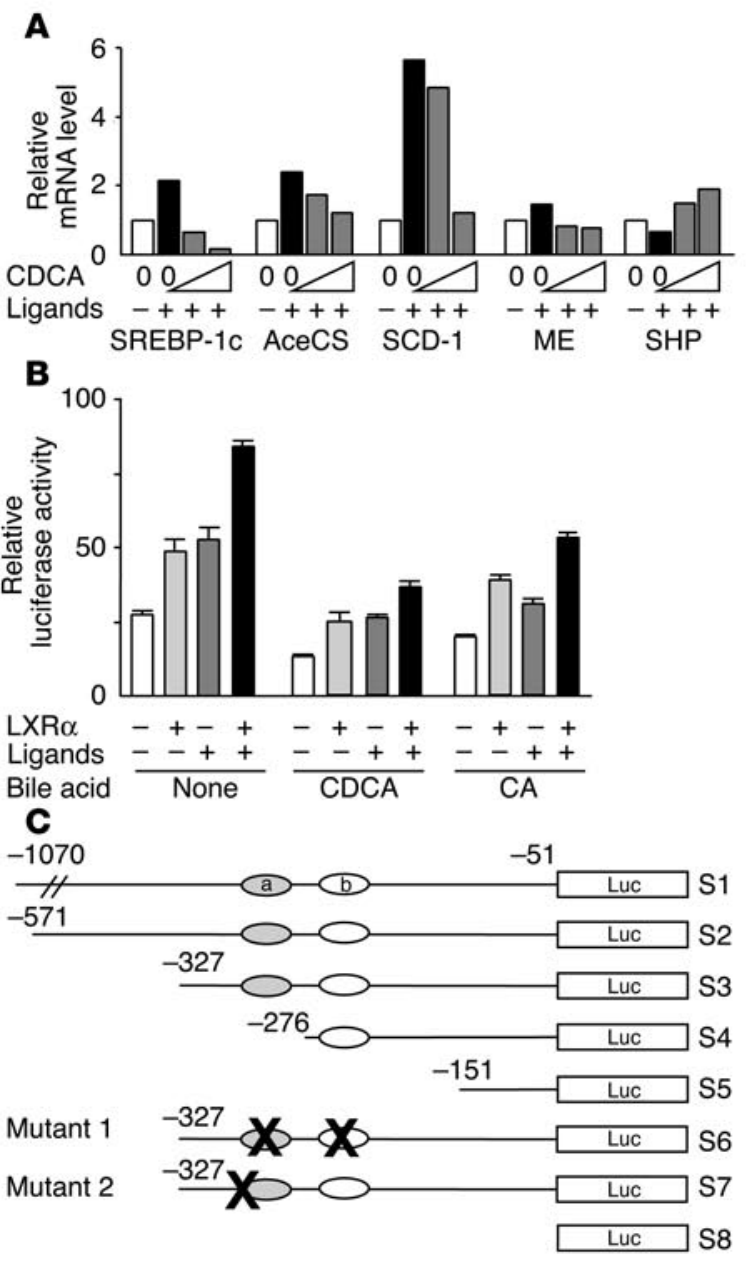

D

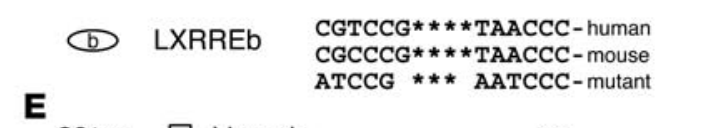

$\mathbf{E}$
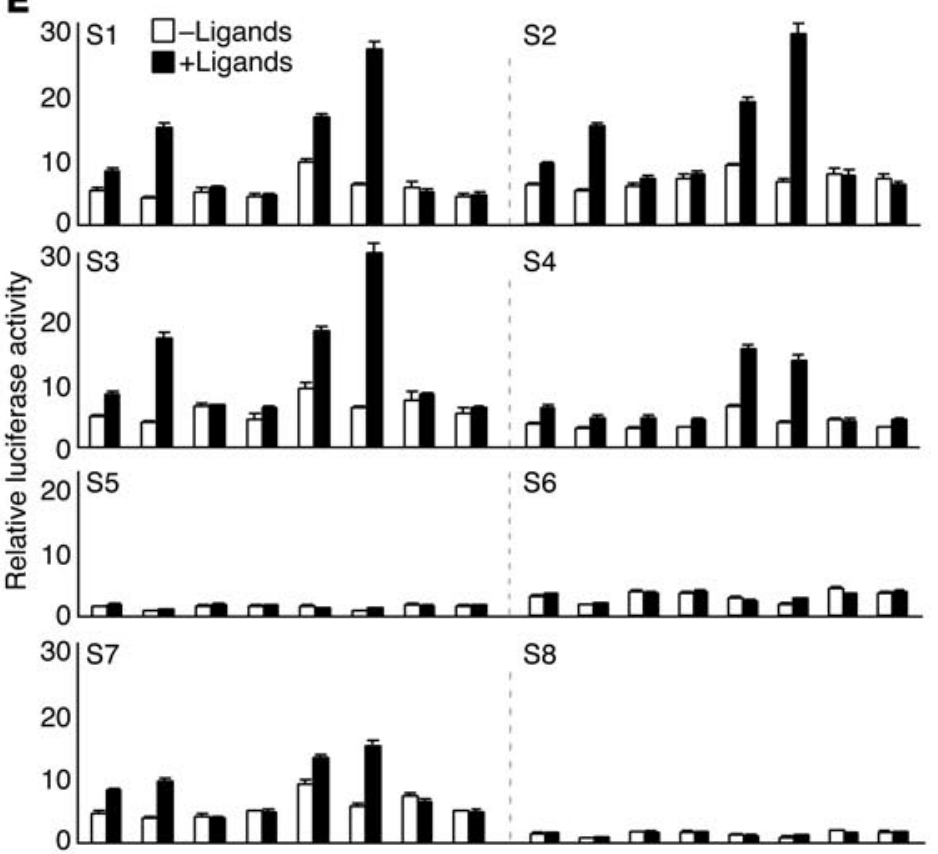

$\mathrm{RXR}$

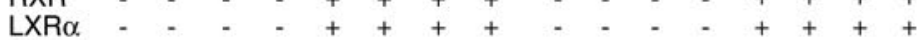

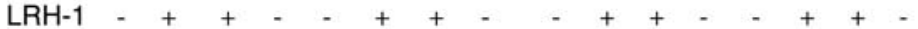
SHP - - + + - - + + - - + + - + +

Figure 3

Bile acids and SHP decrease expression from the SREBP-1c promoter. (A) Expression of SREBP-1c and several of its target genes in mouse liver primary hepatocyte cultures. The presence of ligands for $L X R(22(R)$-hydroxycholesterol, $20 \mu M)$ and $R X R(L G 100268,1 \mu M)$ is indicated by a + sign. FXR was activated by the addition of CDCA to the medium (50 $\mu \mathrm{M}$ and $200 \mu \mathrm{M})$. (B) Activity of the mouse SREBP-1c promoter in the McA-RH7777 cell line after the addition of $200 \mu \mathrm{M}$ CDCA or $200 \mu \mathrm{M}$ CA to the medium. Cells were tested in the absence or presence of cotransfected $L X R \alpha$ and ligands for RXR and LXR at the concentrations specified in A. (C) Schematic representation of the different constructs of the mouse SREBP-1c promoter used in transfection assays. Binding sites for LXR are displayed as ovals. The nucleotide numbering is relative to the SREBP-1c start codon. (D) Sequence comparison of the LXRREs in the human and mouse SREBP-1c promoters. The GenBank accession numbers for the human SREBP-1c promoter sequence are NT_010718 or AC122129. (E) Activity of the mSREBP-1c reporters in McA-RH7777 cells transfected either with an empty expression vector or with the indicated combinations of expression vectors for mouse LRH-1, mouse RXR $\alpha$, human $\mathrm{LXR} \alpha$, mouse SHP in the presence (black bars) or absence (white bars) of LXR (22(R)-hydroxycholesterol; $20 \mu \mathrm{M})$ and RXR agonists (LG100268; $1 \mu \mathrm{M})$. 

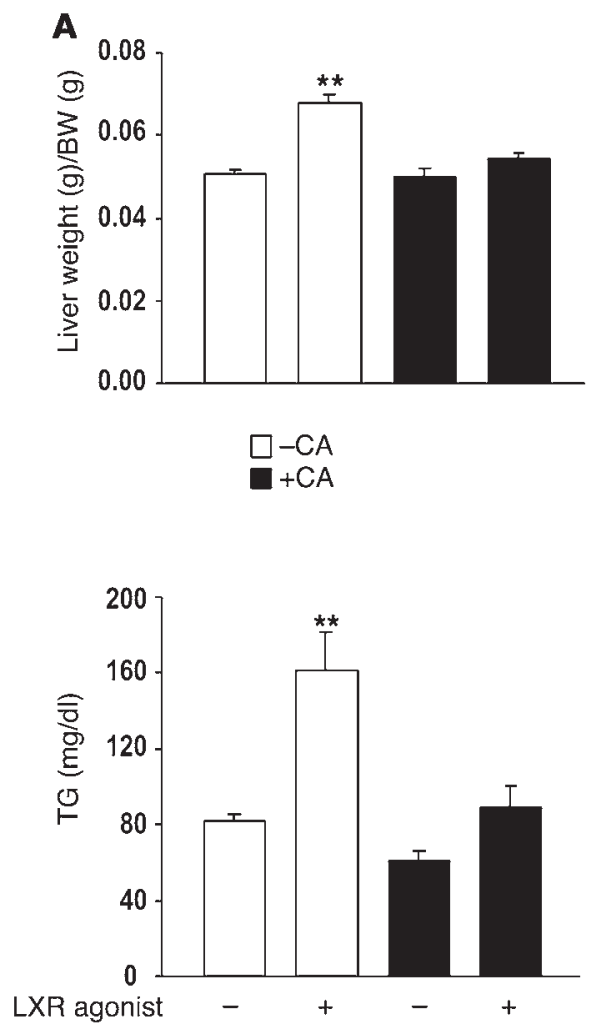
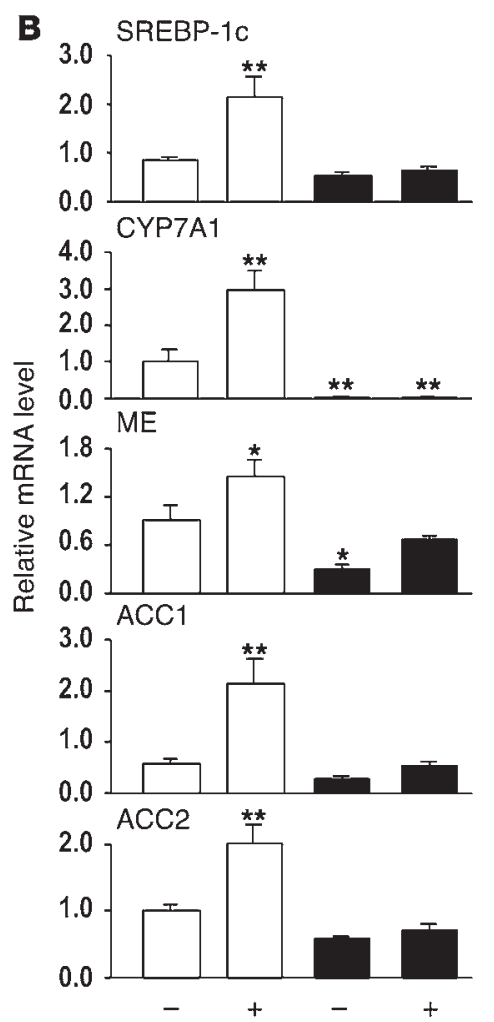
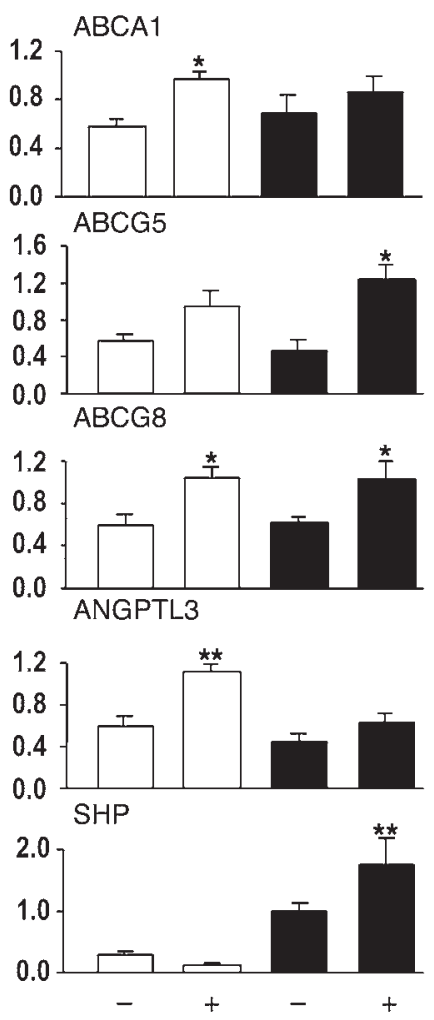

Figure 4

CA attenuates LXR agonist-induced lipogenesis in vivo. (A) Liver weight and serum TGs after feeding a CA-containing diet and coadministration of the LXR agonist T0901317 (age 11 weeks, $n=6$ ). (B) Hepatic expression levels of SREBP-1c, CYP7A1, ME, ACC1, ACC2, ABCA1, ABCG5, ABCG8, ANGPTL3, and SHP as determined using quantitative RT-PCR $(n=4)$.

CA attenuates LXR agonist-induced lipogenesis in vivo. To study the inhibition of the SREBP-1c promoter by CA in more detail, we fed C57BL/6J mice chow or chow supplemented with $0.5 \%$ CA for 1 day. Mice were gavaged with the LXR agonist T0901317 or vehicle, and the different diets were continued for one more day, after which the mice were sacrificed. We confirmed the previously observed induction of liver weight and serum TGs by LXR agonists on a chow diet $(10,11,34)$. Coadministration of CA completely prevented these effects (Figure 4A). As expected, both LXR (SREBP-1c, CYP7A1, ABCA1, ABCG5, ABCG8, and ANGPTL3) and SREBP-1C (ME, ACC1, and ACC2) target genes were induced by administration of the LXR ligand. CA coadministration increased SHP expression and prevented the induction of SREBP-1c and its target genes. Interestingly, some LXR target genes were downregulated by CA (SREBP-1c, CYP7A1, and ANGPTL3), whereas others (ABCA1, ABCG5, and ABCG8) were not (Figure 4B). This led us to conclude that not all LXR target genes are responsive to inhibition by SHP, suggesting that besides LXR there might be additional factor(s) targeted by CA to explain their efficient downregulation.

Attenuation of the TG-lowering effects of FXR agonists in $\mathrm{SHP}^{-/-}$mice. To critically test the role of SHP in the lowering of TG biosynthesis, we administered either a diet containing $0.5 \%$ CA or the synthetic FXR agonist GW4064 to wild-type and SHP-null mice and measured serum TGs after 0,3 , and 7 days. CA and GW4064 significantly lowered serum TGs in the $\mathrm{SHP}^{+/+}$mice. CA seemed more potent in decreasing serum TGs, an effect that may be attributed to the poor pharmacokinetic profile of GW4064. More importantly, this decrease in serum TGs was completely abolished in the $\mathrm{SHP}^{-1-}$ mice (Figure 5A). To determine whether this attenuation of the TG-lowering effects of FXR agonists in $\mathrm{SHP}^{-/-}$mice is paralleled by similar changes at the molecular level, we measured the hepatic expression of SREBP-1c, ME, CYP7A1, and ANGPTL3 in animals sacrificed after a 1-day treatment with the different FXR agonists. Expression of all four genes was significantly reduced in the $\mathrm{SHP}^{+/+}$mice that received CA or GW4064 (Figure 5B). This is in sharp contrast with the results obtained in the $\mathrm{SHP}^{-/-}$mice, in which no decrease in this expression was detected (Figure 5B). The attenuation of CYP7A1 downregulation in $\mathrm{SHP}^{-1-}$ mice upon FXR agonist administration is in apparent contrast with earlier studies (23). This is probably a reflection of the differences in experimental approach (1 day vs. 7 days and $0.5 \%$ CA vs. $1 \%$ CA treatment) that favored activation of secondary regulatory pathways in the earlier studies (23). These data further support that SHP plays an essential role in the SREBP-1c-mediated downregulation of lipogenesis in mice treated with bile acids.

LXR is essential for SHP-mediated lowering of TGs. To determine whether LXR is essential for the TG-lowering effect of bile acids, we used $L X R \alpha / L X R \beta$ double-knockout mice $\left(L X R \alpha / \beta^{-/-}\right)$. Wild-type and $L X R \alpha / \beta^{-/-}$mice were fed with a diet containing $0.5 \%$ CA for 3 days, after which we measured serum TGs (Figure 6A) and hepatic gene expression of SREBP-1c, ME, CYP7A1, and SHP (Figure 6B). As a result of the loss of LXR transactivation in the $L X R \alpha / \beta^{-/-}$mice, the basal level of expression of the LXR (SREBP-1c and CYP7A1) and SREBP-1c (ME) target genes is significantly reduced, causing a lower basal serum TG level (Figure 6, A and B). In wild-type 
A
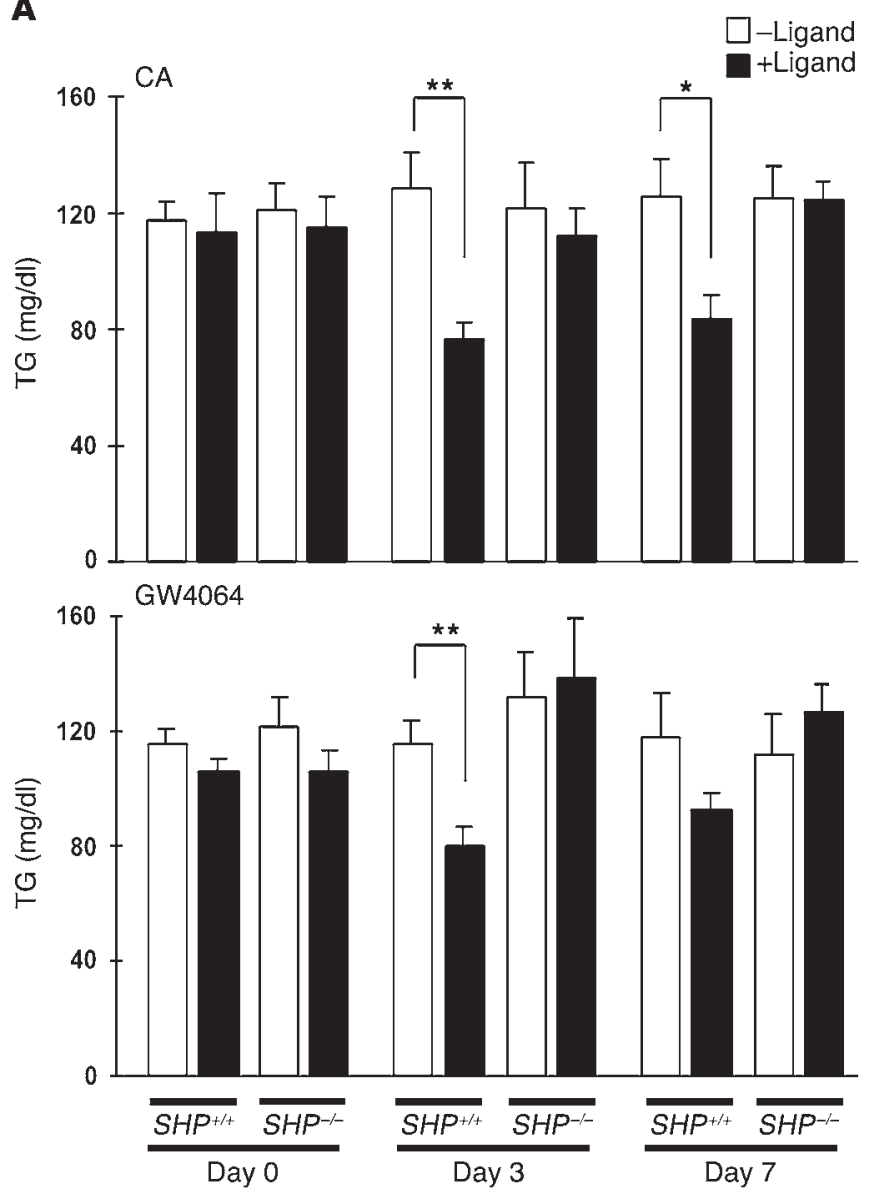

B
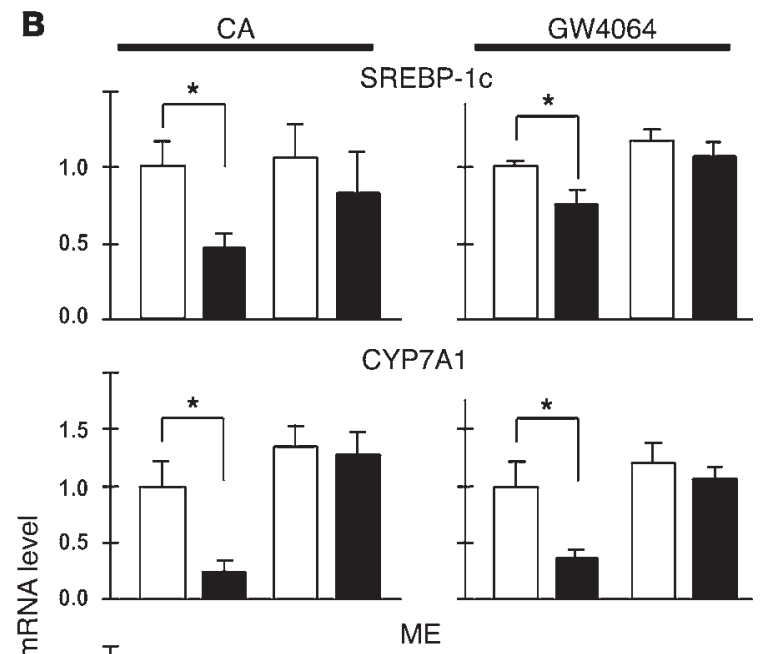

ME
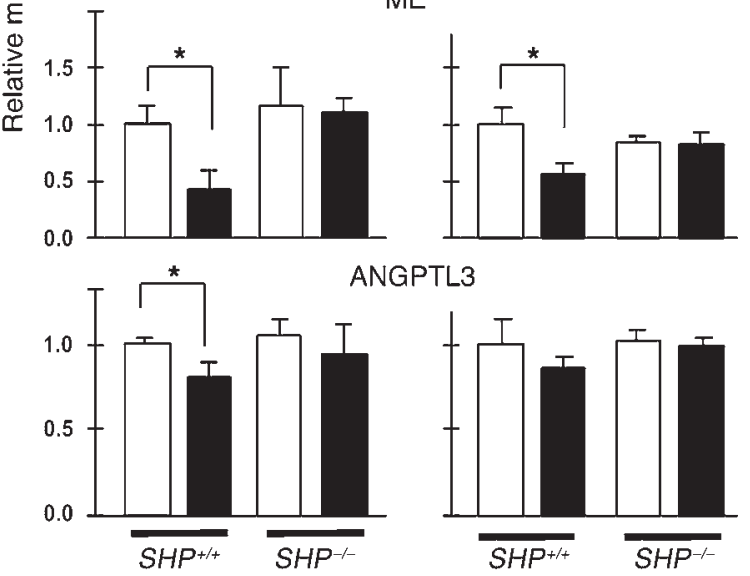

Figure 5

SHP is essential for bile acid-mediated downregulation of lipogenesis. (A) SHP-/- and SHP+/+ mice were fed a diet containing $0.5 \% \mathrm{CA}$ or received GW4064 by oral gavage, and serum TG levels were measured after 0, 3, and 7 days (age 9 weeks, $n=5$ ). (B) In two separate experiments, mice received CA or GW4064 as just described and were sacrificed after 1 day. Hepatic expression levels of SREBP-1c, CYP7A1, ME, and ANGPTL3 were determined using quantitative RT-PCR (age 9 weeks, $n=5$ ).

animals, CA treatment decreased serum TG subsequent to a decrease in the expression SREBP-1c and ME. The decrease in serum TGs, SREBP-1c, and ME is not observed in the $L X R \alpha / \beta^{-/}$ mice (Figure 6, A and B). Though less pronounced in $L X R \alpha / \beta^{-/}$ mice, SHP was induced significantly in both wild-type and $L X R \alpha /$ $\beta^{-/-}$animals. These experiments demonstrate that LXR is essential for the SHP-mediated lowering of TGs in vivo.

\section{Discussion}

We here investigated the effects of bile acids on TG homeostasis and confirmed that bile acids have a beneficial effect in hypertriglyceridemia $(12,15,16,37)$. For these studies, we used KK- $A^{y}$ mice, a model for obesity and type 2 diabetes mellitus. On a normal diet these mice have moderately elevated serum TGs, which increase further upon high-fat feeding. This makes KK- $A^{y}$ mice the first mouse model for diet-induced type IIb and type IV hyperlipoproteinemia. Administration of CA to KK- $A^{y}$ mice reduced serum and hepatic TG concentrations and VLDL secretion in mice fed either a chow or a high-fat diet. The TG-lowering effect was also present in $o b / o b$ mice, another model of obesity, insulin resistance, and hypertriglyceridemia. These results are consistent with earlier studies indicating a reciprocal rela- tionship between bile acid pool and TG production. For example, bile acid-binding resins have been reported to induce the production of VLDL TGs (12-14). In fact, increased TG levels are often an undesirable side effect of the use of these resins to manage lipid disorders. In addition, elevated TGs have recently been reported in patients with CYP7A1 deficiency (22). Whereas mice with a targeted disruption of CYP7A1 do not have a manifest hypertriglyceridemia (38), CYP27-knockout mice, which lack another gene participating in the conversion of cholesterol to bile acids, do have hypertriglyceridemia (39). Interestingly, this elevation of TGs could be attenuated by the addition of bile acids in the diet of the CYP27-knockout mice (39). Furthermore, when compared with normal subjects, hypertriglyceridemic patients have a decreased level of the ileal sodium bile acid transporter, resulting in an impaired enterohepatic recycling of bile acids (40). Finally, addition of bile acids to cultured rat and human hepatocytes decreased VLDL secretion (41-43), underscoring the crucial role of the liver in this process.

Besides lowering serum TGs, CA also lowered serum HDL cholesterol levels in our study. These results are consistent with other recent reports in rodents and humans in which bile acids influence expression of cholesteryl ester transfer protein, phospholipid 
A
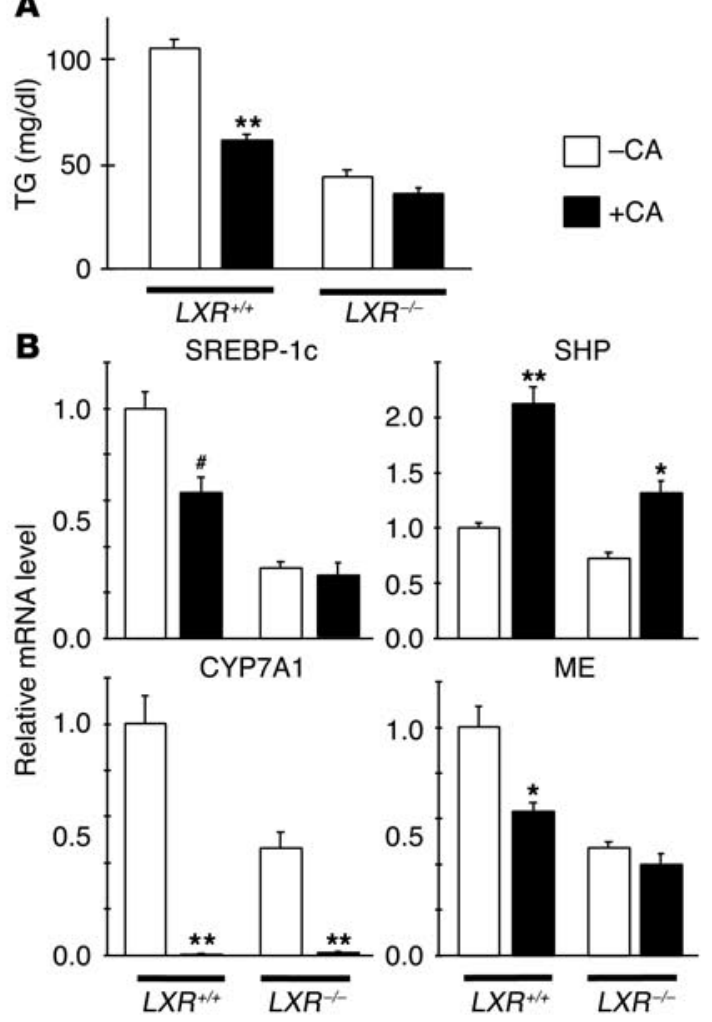

C

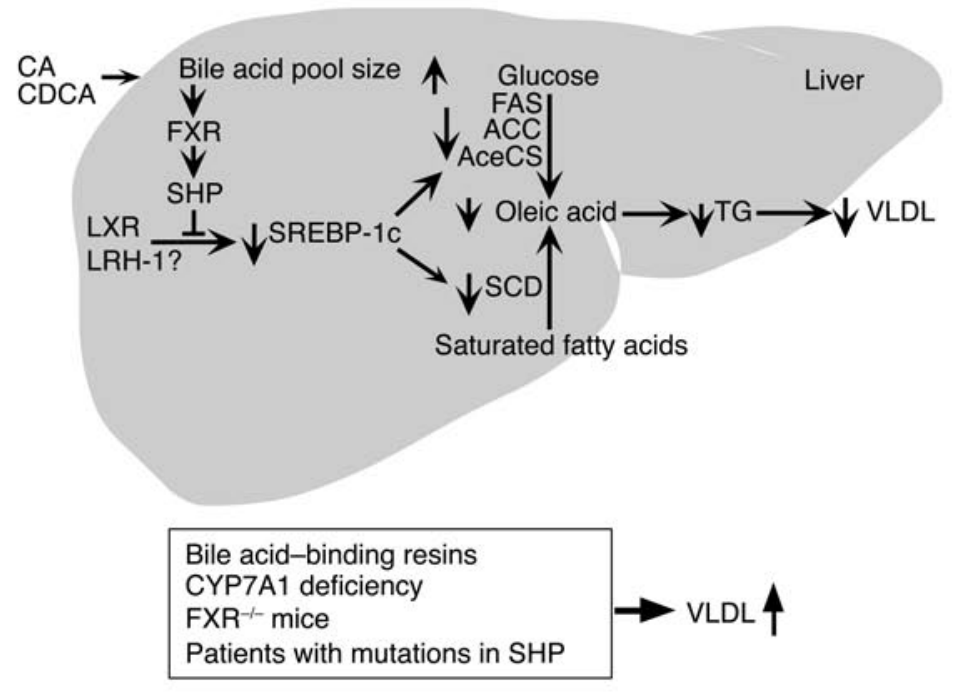

Bile acids (CA, CDCA)
Synthetic FXR ligands (GW4046) $\rightarrow$ VLDL $\downarrow$

Figure 6

LXR is essential for SHP-mediated lowering of TGs. (A) Serum TGs in wild-type and $L X R \alpha / \beta^{-/-}$mice after treatment with $0.5 \%$ CA (age $10-14$ weeks, $n=6$ ). (B) Hepatic expression levels of SREBP-1c, CYP7A1, ME, and SHP in wild-type and $L X R \alpha / \beta^{-/-}$mice after treatment with CA as determined using quantitative RT-PCR. \# denotes $P=0.09(n=6)$. (C) A FXR-SHP-SREBP-1c regulatory cascade. Schematic representation of the proposed role of SHP in mediating the effects of FXR agonists on SREBP-1c expression and lipogenesis.

transfer protein, and ApoA-I $(36,44-46)$. More importantly, the inverse relationship between HDL and TGs, often troublesome in determining whether TGs are an independent risk factor for coronary heart disease, seems uncoupled by CA.

The molecular mechanism for the TG-lowering effects of bile acids has remained elusive. We show here that bile acids lower serum TG levels by targeting SREBP-1c gene expression. Since SREBP-1c is the main regulator of hepatic fatty acid and TG biosynthesis, reducing its expression would be expected to decrease both hepatic TG storage and VLDL production, as we have shown in KK- $A^{y}$ mice. We confirmed here that the basal transcription level of SREBP-1c critically depends on $\operatorname{LXR}(10,11,34)$. We furthermore demonstrate that activation of FXR, by natural and synthetic agonists, increases SHP levels, which in turn reduces SREBP-1c expression. This is in perfect analogy to the mechanism proposed to explain the reduction of CYP7A1 expression by bile acids, which also invoked SHP as a mediator $(17,18,32)$. We validated the central role of SHP in the process of inhibiting SREBP-1c using $\mathrm{SHP}^{-/-}$mice. In these mice, TG levels are not lowered in response to natural (bile acids) and synthetic (GW4064) FXR agonists, which is paralleled at the molecular level by an attenuated response of SREBP-1c and its target gene $\mathrm{ME}$. The combined use of synthetic FXR agonists and $\mathrm{SHP}^{-/-}$mice rules against the possibility that bile acid-activated, but FXR-and SHP-independent, cell signaling pathways are important mediators in the TG-lowering effect $(23,33)$. An important role of LXR as a key player in the regulation of SREBP-1c expression by CA was pro- vided by the lack of a TG-lowering effect subsequent to FXR activation in $L X R \alpha / \beta^{-/-}$mice. The result of the experiment in which we coadministered LXR and FXR agonists, however, showed that the expression of some LXR target genes is decreased in response to CA (SREBP-1c, CYP7A1, and ANGPTL3), while the expression of others is not (ABCA1, ABCG5, and ABCG8), suggesting that besides LXR there is an additional factor targeted by CA to mediate downregulation of these genes. LRH-1 could be that additional factor in view of its role as a competence factor for LXR gene regulation $(17,18$, 36). We were unable, however, to unequivocally confirm or rebut the hypothesis that LRH-1 plays a role in this mechanism because of a total absence of SREBP-1c promoter activity in vitro after mutation of the LXRREs, the absence of a consensus LRH-1RE, and the lack of a mutant LRH-1 mouse model.

The response of SREBP-1c and its target genes to bile acids is strongest after 1 day of treatment and seems to be attenuated after 7 days of treatment. The TG-lowering effects of CA in the KK- $A^{y}$ mice, however, were maintained for up to 3 weeks (data not shown). We believe that SREBP-1c is the initial major target of bile acids to decrease TG levels. The persistence of TG lowering after chronic elevation of the bile acid pool suggests that bile acids could have additional effects on TG homeostasis. Other possible explanations have been put forward that are not mutually exclusive. In cultured human hepatocytes, bile acids induce the expression of PPAR $\alpha$ and its target genes involved in fatty acid $\beta$-oxidation, such as CPT-I (19). We found that CA feeding decreased mRNA levels of genes 
involved in $\beta$-oxidation in C57BL/6J mice, consistent with the previously reported inhibition of PPAR $\alpha$ by bile acids (47). Expression of $\beta$-oxidation genes, furthermore, did not change in KK- $A^{y}$ mice, ruling against a contribution of changes in fatty acid $\beta$-oxidation to explain the decrease in serum TG levels after CA feeding (Table 1). ApoC-II, an activator of lipoprotein lipase, an enzyme that hydrolyzes TGs from VLDL and chylomicrons and as such could lower serum TG levels, has been reported to be an FXR target gene (20). In addition, we show here that, similar to SREBP-1c, the expression of the LXR target gene ANGPTL3 is lowered by CA feeding via a SHP-dependent mechanism. ANGPTL3 can increase serum TGs by inactivating lipoprotein lipase (48). Thus besides reducing hepatic VLDL production via effects on SREBP-1c, bile acids will also increase VLDL clearance via effects on ApoC-II and ANGPTL3. Very recently, Zhang et al. reported that FXR mRNA levels are increased by PPAR $\gamma$ and HNF $4 \alpha$. These investigators further show that PGC$1 \alpha$ is a coactivator for FXR (49). Fasting markedly induces hepatic PGC- $1 \alpha$ levels. Under this condition, PGC- $1 \alpha$ and FXR cooperate to maintain energy homeostasis by decreasing serum TG levels via effects on VLDL clearance (ApoC-II, reducing hepatic TG biosynthesis and secretion via SREBP-1c, and by increasing fatty acid $\beta$-oxidation via PGC- $1 \alpha(49)$.

At the metabolite level, bile acids reduce their biosynthesis from cholesterol, as reflected by the downregulation of CYP7A1, increasing hepatic cholesterol (Figure 2C) and oxysterol levels. This will, on the one hand, activate LXR and induce SREBP-1c expression as a compensation for its repression by SHP. Such an LXR activation is reflected by the tendency of ABCA1 and ABCG5 expression to increase after bile acid feeding (Table 1). On the other hand, and perhaps more importantly to explain the long-term effects, elevated cholesterol levels will decrease SREBP processing, consequently reducing the expression of SREBP target genes. The reduced expression of genes involved in cholesterol uptake (LDL-R) and biosynthesis (HMG-CoA reductase and synthase) are consistent with a decreased activity of the SREBP-2 pathway. Likewise, the persistent decrease in some lipogenic genes (AceCS and ME), despite almost normal SREBP-1c mRNA levels 7 days after CA feeding, would be in agreement with such an effect on SREBP processing. Although our data prove that the SREBP-1c gene is the major initial target of bile acids to decrease TG levels, they also warrant further detailed exploration to elucidate the molecular pathways underlying the chronic TG-lowering effects of bile acids.

Since most mouse strains are very resistant to food-induced hyperlipoproteinemia, expression of the active form of SREBP-1c in the liver did not increase serum TGs despite increased hepatic TG synthesis (7). Several other lines of evidence, however, point toward an important role for liver SREBP-1c in lipogenesis, obesity, and hyperlipidemia. Hepatic SREBP-1c gene expression is increased in animal models of obesity $(50,51)$ and type 2 diabetes $(52,53)$. In addition, obese patients have an increased hepatic lipogenesis (and often a subsequent hypertriglyceridemia) that could contribute to hepatic insulin resistance and their excessive fat mass, which decreased after energy restriction (54). Data on the SREBP-1C (55), SCAP (56), and S1P (57) knockout mice further prove that the SREBP pathway is important for TG synthesis in the liver and the determination of serum TG levels.

The FXR-SHP-SREBP-1c regulatory cascade that we propose (Figure 6C) can explain a number of previous results linking FXR activity to TG levels in liver and serum. $F X R^{-/-}$mice show decreased basal expression of SHP and have increased serum and hepatic TG levels (31). Moreover, plasma TG decreased when rats were treated for 7 days with the synthetic FXR ligand GW4046 (58). Earlier studies had already shown that the profound lowering of hepatic TGs, observed with a CA diet, was significantly diminished in the $\mathrm{SHP}^{-/-}$mice. In these $\mathrm{SHP}^{-/-}$mice, SREBP-1 expression was increased relative to $\mathrm{SHP}^{+/+}$animals when both were fed a diet containing CA (23). Another study showed that the expression of the lipogenic genes ACC, GPAT, and FAS was increased in the SHPdeficient animals (33). These effects with two independently derived $\mathrm{SHP}^{-/-}$lines are consistent with the direct repressive effect of SHP in SREBP-1c expression described here. This effect is also consistent with the association of hypertriglyceridemia (and mild obesity) with mutations in the SHP gene in Japanese patients (59). We conclude that the acute attenuation of the lipogenic activity of SREBP-1c by SHP induction can explain the inhibitory effects of bile acids on TG production. We therefore suggest that strategies aimed at increasing FXR activity and the repressive effects of SHP on SREBP-1c activity should be explored as a novel approach to correct hypertriglyceridemia. The use of bile acids such as CA and CDCA is limited in humans because they can cause significant hepatotoxicity and raise LDL cholesterol by bringing about a decrease in LDL-R activity (60), an effect that was also observed in our mouse studies. It might, however, be possible to develop FXR modulators that retain the beneficial effects on hepatic TG biosynthesis, but lack the LDLraising potential of bile acids like CA and CDCA.

\section{Acknowledgments}

Work in the laboratories of the authors is supported by grants of CNRS, INSERM, ULP, Hôpital Universitaire de Strasbourg, NIH (1P01 DK59820-01), EMBO, and the EU (QLG1CT-1999-00674 and QLRT-2001-00930). D.J. Mangelsdorf is an investigator and A. Moschetta is an associate of the Howard Hughes Medical Institute, which partly funded this work. The authors thank Pierre Chambon, Elisabeth Fayard, Osamu Ezaki, and Jurgen Lehmann for helpful discussions, and Marie-France Champy and the staff of the Institut Clinique de la Souris for technical assistance.

Received for publication January 9, 2004, and accepted in revised form March 23, 2004.

Address correspondence to: Johan Auwerx, Institut de Génétique et Biologie Moléculaire et Cellulaire, 1 Rue Laurent Fries, Parc d'Innovation, 67404 Illkirch, France. Phone: 33-388653425; Fax: 33-388653201; E-mail: auwerx@igbmc.u-strasbg.fr.
1. Cullen, P. 2000. Evidence that triglycerides are an independent coronary heart disease risk factor. $\mathrm{Am}$. J. Cardiol. 86:943-949.

2. Ginsberg, H.N. 2001. Hypertriglyceridemia: new insights and new approaches to pharmacologic therapy. Am. J. Cardiol. 87:1174-1180.

3. Schoonjans, K., Staels, B., and Auwerx, J. 1996. Role of the peroxisome proliferator-activated receptor (PPAR) in mediating the effects of fibrates and fatty acids on gene expression. J. Lipid Res. 37:907-925.

4. Horton, J.D., Goldstein, J.L., and Brown, M.S. 2002. SREBPs: activators of the complete program of cholesterol and fatty acid synthesis in the liver. J. Clin. Invest. 109:1125-1131. doi:10.1172/ JCI200215593.

5. Brown, M.S., and Goldstein, J.L. 1997. The SREBP pathway: regulation of cholesterol metabolism by proteolysis of a membrane-bound transcription factor. Cell. 89:331-340.

6. Shimano, H., et al. 1996. Overproduction of cholesterol and fatty acids causes massive liver enlargement in transgenic mice expressing truncated SREBP-1a. J. Clin. Invest. 98:1575-1584.

7. Shimano, H., et al. 1997. Isoform 1c of sterol regulatory element binding protein is less active than isoform 1a in livers of transgenic mice and in cultured cells. J. Clin. Invest. 99:846-854. 
8. Foretz, M., et al. 1999. ADD1/SREBP-1c is required in the activation of hepatic lipogenic gene expression by glucose. Mol. Cell. Biol. 19:3760-3768.

9. Shimomura, I., et al. 1999. Insulin selectively increases SREBP-1c mRNA in the livers of rats with streptozotocin-induced diabetes. Proc. Natl. Acad. Sci. U. S. A. 96:13656-13661.

10. Repa, J.J., et al. 2000. Regulation of mouse sterol regulatory element-binding protein-1c gene (SREBP-1c) by oxysterol receptors, LXR $\alpha$ and LXR $\beta$. Genes Dev. 14:2819-2830.

11. Schultz, J.R., et al. 2000. Role of LXRs in control of lipogenesis. Genes Dev. 14:2831-2838.

12. Angelin, B., Einarsson, K., Hellstrom, K., and Leijd, B. 1978. Effects of cholestyramine and chenodeoxycholic acid on the metabolism of endogenous triglyceride in hyperlipoproteinemia. J. Lipid Res. 19:1017-1024

13. Beil, U., Crouse, J.R., Einarsson, K., and Grundy, S.M. 1982. Effects of interruption of the enterohepatic circulation of bile acids on the transport of very low density-lipoprotein triglycerides. Metabolism. 31:438-444.

14. Crouse, J.R. 1987. Hypertriglyceridemia: a contraindication to the use of bile acid binding resins. Am. J. Med. 83:243-248.

15. Bateson, M.C., Maclean, D., Evans, J.R., and Bouchier, I.A. 1978. Chenodeoxycholic acid therapy for hypertriglyceridaemia in men. Br. J. Clin. Pharmacol. 5:249-254.

16. Carulli, N., et al. 1981. Chenodeoxycholic acid and ursodeoxycholic acid effects in endogenous hypertriglyceridemias. A controlled double-blind trial. J. Clin. Pharmacol. 21:436-442.

17. Goodwin, B., et al. 2000. A regulatory cascade of the nuclear receptors FXR, SHP-1, and LRH-1 represses bile acid biosynthesis. Mol. Cell. 6:517-526

18. Lu, T.T., et al. 2000. Molecular basis for feedback regulation of bile acid synthesis by nuclear receptors. Mol. Cell. 6:507-515.

19. Pineda Torra, I., et al. 2003. Bile acids induce the expression of the human peroxisome proliferator-activated receptor $\alpha$ gene via activation of the farnesoid X receptor. Mol. Endocrinol. 17:259-272.

20. Kast, H.R., et al. 2001. Farnesoid X-activated receptor induces apolipoprotein C-II transcription: a molecular mechanism linking plasma triglyceride levels to bile acids. Mol. Endocrinol. 15:1720-1728.

21. Beigneux, A., Hofmann, A.F., and Young, S.G. 2002 Human CYP7A1 deficiency: progress and enigmas. J. Clin. Invest. 110:29-31. doi:10.1172/JCI200216076.

22. Pullinger, C.R., et al. 2002. Human cholesterol $7 \alpha$ hydroxylase (CYP7A1) deficiency has a hypercholesterolemic phenotype. J. Clin. Invest. 110:109-117. doi:10.1172/JCI200215387

23. Wang, L., et al. 2002. Redundant pathways for negative feedback regulation of bile acid production. Dev. Cell. 2:721-731.

24. Rocchi, S., et al. 2001. A unique PPAR $\gamma$ ligand with potent insulin-sensitizing yet weak adipogenic activity. Mol. Cell. 8:737-747.

25. Picard, F., et al. 2002. SRC-1 and TIF2 control energy balance between white and brown adipose tissues. Cell. 111:931-941.

26. Boisfer, E., et al. 1999. Overexpression of human apolipoprotein A-II in mice induces hypertriglyceridemia due to defective very low density lipopro- tein hydrolysis. J. Biol. Chem. 274:11564-11572.

27. Nakamura, M. 1962. A diabetic strain of the mouse. Proc. Jpn. Acad. 38:348-352.

28. Yen, T.T., Gill, A.M., Frigeri, L.G., Barsh, G.S., and Wolff, G.L. 1994. Obesity, diabetes, and neoplasia in yellow $\mathrm{A}(\mathrm{vy})^{/-}$mice: ectopic expression of the agouti gene. FASEB J. 8:479-488.

29. Nishimura, M. 1969. Breeding of mice strains for diabetes mellitus. Exp. Anim. 18:147-157.

30. Ikemoto, et al. 1997. Cholate inhibits high-fat diet-induced hyperglycemia and obesity with acylCoA synthetase mRNA decrease. Am. J. Physiol. 273:E37-E45.

31. Sinal, C.J., et al. 2000. Targeted disruption of the nuclear receptor FXR/BAR impairs bile acid and lipid homeostasis. Cell. 102:731-744

32. Brendel, C., Schoonjans, K., Botrugno, O.A., Treuter, E., and Auwerx, J. 2002. The small heterodimer partner interacts with the liver $\mathrm{X}$ receptor $\alpha$ and represses its transcriptional activity. Mol. Endocrinol. 16:2065-2076.

33. Kerr, T.A., et al. 2002. Loss of nuclear receptor SHP impairs but does not eliminate negative feedback regulation of bile acid synthesis. Dev. Cell. 2:713-720.

34. Yoshikawa, T., et al. 2001. Identification of liver X receptor-retinoid $\mathrm{X}$ receptor as an activator of the sterol regulatory element-binding protein $1 \mathrm{c}$ gene promoter. Mol. Cell. Biol. 21:2991-3000.

35. Goodwin, B., et al. 2003. Differential regulation of rat and human CYP7A1 by the nuclear oxysterol receptor liver X receptor- $\alpha$. Mol. Endocrinol. 17:386-394

36. Luo, Y., Liang, C.P., and Tall, A.R. 2001. The orphan nuclear receptor LRH-1 potentiates the sterolmediated induction of the human CETP gene by liver X receptor. J. Biol. Chem. 276:24767-24773.

37. Iser, J.H., and Sali, A. 1981. Chenodeoxycholic acid: a review of its pharmacological properties and therapeutic use. Drugs. 21:90-119.

38. Schwarz, M., et al. 1996. Disruption of cholesterol $7 \alpha$-hydroxylase gene in mice. II. Bile acid deficiency is overcome by induction of oxysterol $7 \alpha$-hydroxylase. J. Biol. Chem. 271:18024-18031.

39. Repa, J.J., et al. 2000. Disruption of the sterol 27hydroxylase gene in mice results in hepatomegaly and hypertriglyceridemia. Reversal by cholic acid feeding. J. Biol. Chem. 275:39685-39692.

40. Duane, W.C., Hartich, L.A., Bartman, A.E., and Ho, S.B. 2000. Diminished gene expression of ileal apical sodium bile acid transporter explains impaired absorption of bile acid in patients with hypertriglyceridemia. J. Lipid Res. 41:1384-1389.

41. del Pozo, R., and Barth, C.A. 1987. Bile acids inhibit secretion of very low density lipoprotein by rat hepatocytes. Biol. Chem. Hoppe-Seyler. 368:887-893.

42. Lin, Y., et al. 1996. Characterization of the inhibitory effects of bile acids on very-low-density lipoprotein secretion by rat hepatocytes in primary culture. Biochem. J. 316:531-538.

43. Lin, Y., et al. 1996. Bile acids suppress the secretion of very-low-density lipoprotein by human hepatocytes in primary culture. Hepatology. 23:218-228.

44. Srivastava, R.A., Srivastava, N., and Averna, M. 2000 Dietary cholic acid lowers plasma levels of mouse and human apolipoprotein A-I primarily via a transcriptional mechanism. Eur. J. Biochem. 267:4272-4280.
45. Urizar, N.L., Dowhan, D.H., and Moore, D.D. 2000. The farnesoid $\mathrm{X}$-activated receptor mediates bile acid activation of phospholipid transfer protein gene expression. J. Biol. Chem. 275:39313-39317

46. Claudel, T., et al. 2002. Bile acid-activated nuclear receptor FXR suppresses apolipoprotein A-I transcription via a negative FXR response element. J. Clin. Invest. 109:961-971. doi:10.1172/ JCI200214505

47. Sinal, C.J., Yoon, M., and Gonzalez, F.J. 2001. Antagonism of the actions of peroxisome proliferator-activated receptor- $\alpha$ by bile acids. J. Biol. Chem. 276:47154-47162.

48. Inaba, T., et al. 2003. Angiopoietin-like protein 3 (Angptl3) mediates hypertriglyceridemia induced by the liver X receptor. J. Biol. Chem. 278:21344-21351.

49. Zhang, Y., Castellani, L.W., Sinal, C.J., Gonzalez, F.J., and Edwards, P.A. 2004. Peroxisome proliferator-activated receptor- $\gamma$ coactivator $1 \alpha$ (PGC-1 $\alpha$ ) regulates triglyceride metabolism by activation of the nuclear receptor FXR. Genes Dev. 18:157-169.

50. Shimomura, I., et al. 2000. Decreased IRS-2 and increased SREBP-1c lead to mixed insulin resistance and sensitivity in livers of lipodystrophic and ob/ob mice. Mol. Cell. 6:77-86.

51. Elam, M.B., et al. 2001. Increased hepatic VLDL secretion, lipogenesis, and SREBP-1 expression in the corpulent JCR:LA-cp rat. J. Lipid Res. 42:2039-2048.

52. Shimomura, I., Bashmakov, Y., and Horton, J.D. 1999. Increased levels of nuclear SREBP-1c associated with fatty livers in two mouse models of diabetes mellitus. J. Biol. Chem. 274:30028-30032.

53. Tobe, K., et al. 2001. Increased expression of the sterol regulatory element-binding protein-1 gene in insulin receptor substrate- $2^{(-/)}$mouse liver. J. Biol. Chem. 276:38337-38340.

54. Diraison, F., Dusserre, E., Vidal, H., Sothier, M., and Beylot, M. 2002. Increased hepatic lipogenesis but decreased expression of lipogenic gene in adipose tissue in human obesity. Am. J. Physiol. Endocrinol. Metab. 282:E46-E51.

55. Liang, G., et al. 2002. Diminished hepatic response to fasting/refeeding and liver $\mathrm{X}$ receptor agonists in mice with selective deficiency of sterol regulatory element-binding protein-1c. J. Biol. Chem. 277:9520-9528.

56. Matsuda, M., et al. 2001. SREBP cleavage-activating protein (SCAP) is required for increased lipid synthesis in liver induced by cholesterol deprivation and insulin elevation. Genes Dev. 15:1206-1216.

57. Yang, J., et al. 2001. Decreased lipid synthesis in livers of mice with disrupted Site-1 protease gene. Proc. Natl. Acad. Sci. U. S. A. 98:13607-13612.

58. Maloney, P.R., et al. 2000. Identification of a chemical tool for the orphan nuclear receptor FXR. J. Med. Chem. 43:2971-2974.

59. Nishigori, H., et al. 2001. Mutations in the small heterodimer partner gene are associated with mild obesity in Japanese subjects. Proc. Natl. Acad. Sci. U. S. A. 98:575-580.

60. Schoenfield, L.J., and Lachin, J.M. 1981. Chenodiol (chenodeoxycholic acid) for dissolution of gallstones: the National Cooperative Gallstone Study. A controlled trial of efficacy and safety. Ann. Intern. Med. 95:257-282. 